\title{
Redefining How Neutrinos Impede Dark Matter Searches
}

\author{
A new definition of the "neutrino floor" in dark matter experiments \\ clarifies the challenges ahead in differentiating neutrinos from WIMPs. \\ By Christopher Crockett
}

$\mathrm{M}$ any underground labs are searching for dark matter in the form of weakly interacting massive particles (WIMPs). As the sensitivities of these experiments improve, they will have to contend with neutrinos, which can leave observational signatures remarkably like those predicted for dark matter. This challenge has prompted the idea of a "neutrino floor," a theoretical limit on the types of dark matter particles that could be discovered (see Research News: Neutrinos Rising from the Floor). Now, Ciaran O'Hare of the University of Sydney proposes a new definition of the neutrino floor that is more statistically meaningful than previous calculations and does not depend on arbitrary experimental parameters [1].

Hunts for WIMPs rely on spotting atomic nuclei scattered by dark matter particles. Unfortunately, neutrinos can also scatter atomic nuclei, producing similar signals. The neutrino floor is usually defined as the point at which the dark matter signal gets buried in the neutrino signal. But the floor isn't a hard limit-it depends on uncertainties in the neutrino flux and can be

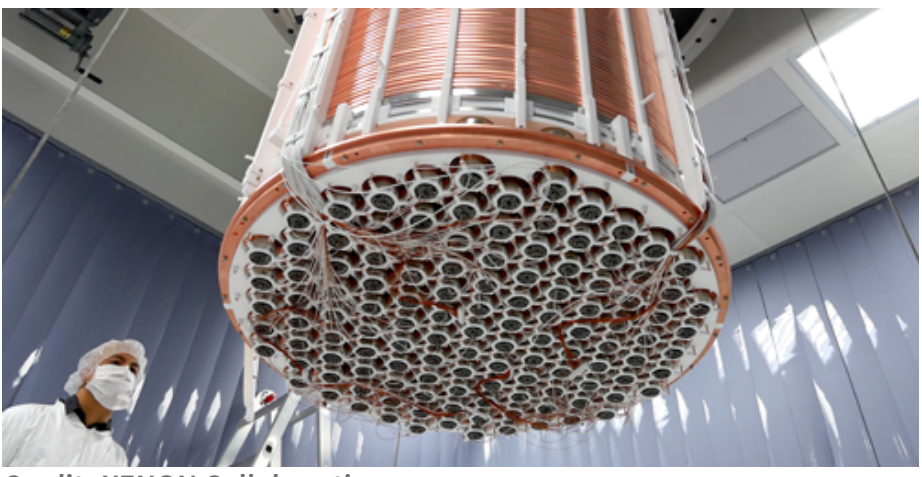

Credit: XENON Collaboration overcome by detecting lots of events. This has led some to talk about a "neutrino fog," a region of discovery space where differentiating dark matter from neutrinos is hard but not impossible.

O'Hare's new definition of the neutrino floor puts it at the edge of this fog. Relying on observed and calculated neutrino fluxes from many sources as well as a common dark matter model, it marks the point at which any dark matter experiment will start to be limited by the neutrino background. While paralleling previous calculations, the new boundary predicts that low- and high-mass WIMPs must scatter nuclei at a higher rate than previously thought to be discerned against the fog, while intermediate-mass WIMPs have a bit more leeway.

Christopher Crockett is a freelance writer based in Arlington, Virginia.

\section{REFERENCES}

1. C. A. J. O'Hare, "New definition of the neutrino floor for direct dark matter searches," Phys. Rev. Lett. 127, 251802 (2021). 